\title{
AGENT-BASED TECHNOLOGIES IN DEFENSE AND SECURITY
}

$\mathrm{T}$ he field of autonomous agents and multi-agent systems is an exciting and rapidly expanding area of research and development. In the last few years, there has been a growing interest in the application of agent-based systems to various securityrelated and military domains. In this special issue of Information \& Security we shall present the results achieved in this area, discuss the benefits (and drawbacks) that agent-based systems may bring to the military and the broader security community, and provide a list of research and practical challenges that should be tackled in the near future so that the full potential of agent-based systems is realized.

Published results reflect applications, or potential applications, of agent-based systems for situational assessment for submariners; agents that discover and alert on conflicts in air mobility plans; software agents that aid military intelligence efforts; cooperating agents that address the problem of discovering and prosecuting mobile, time-critical targets; multi-agent systems for mechanics' decision support during aircraft maintenance; software systems that support mission critical team decisionmaking; multi-agent systems that assist defense acquisition and contracting; integrated marine multi-agent $\mathrm{C} 2$ system providing "near" real-time situation awareness; multi-agent simulations for planning and executing joint operations, rehearsing dangerous tactical operations, noncombatant evacuation operation and logistics planning; multi-agent system for identifying and exploiting emergent collective behavior on the battlefield; agents that recognize aircraft maneuvers during simulated flight; intelligent agents for threat assessment in small unit operations; multi-agent intelligence analysis systems; Internet-based multi-agent systems for military training; robotic demining agents; multi-agent system that handle tank platoon movement.

During application researchers encounter a set of methodological and technical problems. For example, there are no universally accepted standard ontologies and semantic definition languages in the military; it is necessary to improve quality and efficiency of multi-agent simulations of operations; it is problematic to integrate agent 
technology in existing large-scale military simulation system; there is a need to develop robust agent behavior control mechanisms. Last but not least, is the challenge to prove to the military the utility and reliability of agents. Nevertheless, the trend is gradual transition from limited applications performing isolated functions to collaborative applications interacting with other systems and data sources to reach complex goals.

\section{Objectives of the special issue}

The purpose of this special issue is to present latest achievements and new ideas in applying agents and multi-agent systems to the military and the broader security domain. The topics include but are not limited to:

- General and specific architectures of agents in different settings and environments

- Cooperation and competition; coordination and collaboration

- Negotiation, consensus development, conflict detection and resolution

- Communication protocols and languages (communication standards)

- Intelligent cognitive activities jointly realized by multiple agents, e.g., distributed problem solving, planning, learning, and decision making

- Emergent behavior and organizational intelligence

- Organizational structuring and dynamics

- Mobile agents as general-purpose framework for distributed applications

- Performance issues; security, reliability, and robustness

- Agents and the interoperability of heterogeneous systems

- Human-agent interaction and interfaces

- Architectures, environments and languages for mobile and secure information services

- Agent capability requirements in military applications

- Analysis of experience in implementing and operating agent-based systems

In particular, based on the accepted publications, we have decided to organize the special journal issue on Agent-based Technologies in two numbers. Volume 8, number 1 focuses on the following two groups of topics:

- Agent -based Defense Modeling and Simulation

- Security of Agent-based Systems. 
Number 2 deals with:

- Coalition Operations Planning and Negotiations

- Agents in Resource Planning

\section{Agent -based Defense Modeling and Simulation}

Computer simulation is a valuable tool for complex decision-making, especially in military and civilian operations in the land, air or sea. Simulation has been used in the military domain for the evaluation of acquisitions, missions and force development options. Modeling and simulation for this purpose is becoming increasingly complex as multi-role, multi-platform and multi-system aspects are taken into consideration. The complexity of this task is further increased by the difficulty in modeling human decision-making using conventional software approaches. Current implementations of computer generated forces have proven to be very useful, but do not model human reasoning and cannot easily model team behavior. Applications of intelligent agents in military simulations have proved highly effective. This is due to the capability of agents to represent individual reasoning and from the architectural advantages of that representation to the user due to the ease of setting up and modifying operational reasoning or tactics for various studies. In addition, intelligent agents extend the modeling of reasoning to explicitly model the communications and coordination of activities required for team behavior.

The aim of the first group of papers is to point out the importance of agent-based modeling and simulation, as a scientific concept and technological possibility, to enhance the potential of simulation in both civilian and defense applications.

Agent-based distillations represent an emerging technology within the field of combat modeling and simulation. They utilize agent-based methods to model combat as a complex adaptive system. Existing distillations have already shown the great promise of this approach in providing analysts with valuable insights into areas such as nonlinearity, co-evolving landscapes and intangibles.

The paper by Michael Barlow and Adam Easton present a new multi-agent-based distillation known as CROCADILE - a Comprehensive, Research Oriented, Combat Agent Distillation Implemented in the Littoral Environment. This is a new distillation system that significantly extends the agent-based distillation field. CROCADILE has been designed as an open distillation harness and developed at the Australian Defense Force Academy.

Among the key new features that CROCADILE brings to agent-based distillations are the following: a true 3D world, probabilistic or projectile-physics combat resolution, extensible object-oriented design, multiple agent control paradigms, sophisticated 
command, mission, and communication structures for agents, higher fidelity combat resolution models that incorporate blast effects, round penetration, rates of fire, and line of site, and multi-team structure including neutrals and levels of enmity/alliance and communication between teams.

The paper details the object-oriented design together with the various efficiencies employed to deliver a real-time 3D implementation involving hundreds of agents. The system and its explanation are illustrated via a scenario examining a possible structure for the Australian Army's "Army after Next" concept.

The emphasis on timely, accurate information in modern warfare, and the availability of modern communications, has led to the development of increasingly complex command and control systems. It is important to understand the behavior of these systems under a variety of circumstances. However, as they are difficult to analyze manually, advanced modeling and simulation tools for command and control systems development are required. As we have already elaborated, the challenge in these systems is to model the reasoning associated with different roles in the hierarchy. Intelligent agents can represent the reasoning and command capabilities associated with their assigned roles in the hierarchy, allowing different command and control strategies to be quickly evaluated under varying circumstances.

These intelligent agent simulation models are in the focus of the second paper. Such agent models consist of a number of entities which interact locally in order to produce global emergent behavior. In complex systems, elaborate and unpredictable properties arise from the interaction of the constituents. Examples of such emergent properties include how the system organizes itself, how it finds a balance between order and disorder, and how agents, both individually and collectively, evolve new behaviors in response to change. Some of the emergent behaviors can be surprising, and it is the aim of the work described by James Moffat and Susan Witty to produce a theory of such processes which helps to explain the types of behavior to be expected.

In a nutshell, understanding the behavior of such agent-based combat models is now becoming more important, especially as the agents gain intelligence and try to outsmart each other, producing potentially very complex behavior. The principal variables in these models can often be separated out from the rest of the model to produce a metamodel that is aimed at decreasing the run-time of the original model while still retaining the characteristics and arriving at the same final solution as the original model.

In their paper, James Moffat and Susan Witty discuss the development of such a metamodel of an intelligent agent simulation model. Their metamodel is a mathematical abstraction of such a simulation, composed of two parts. For the first part, the fractal dimension of a force is introduced as a parameter measuring the 
emergent ability of such forces to cluster locally, corresponding to local decisionmaking by individual agents. For the second part the authors consider the mathematics of Bayesian Decision-Making as a metamodel for top down decision processes in such simulation models.

\section{Security of Agent-based Systems}

A great deal of military applications is characterized by an environment that is distributed, heterogeneous, dynamic, unpredictable, insecure, and unreliable. More specifically, the latency and bandwidth of network connections varies enormously in space and time, especially with the growing demand for wireless networks, satellite connections, mobile devices (laptops and personal digital assistants), and other highly volatile communications networks. Therefore, computing paradigms that are robust in the presence of network volatility are largely needed. The applications in this environment must be able to adapt to different and changing conditions.

Mobile (transportable) agents are an excellent computing mechanism to realize such applications, especially when used in a wireless environment. These mobile agents have the potential to provide a convenient, efficient and robust programming paradigm for distributed applications. Mobile agency is also the paradigm that has been suggested for allowing efficient access to remote resources. It is particularly attractive in situations where the amount of information to be processed is large relative to the network bandwidth.

A mobile/transportable agent is an autonomous software program/entity that can migrate from host to host in a heterogeneous network. The program chooses when and where to migrate. It can suspend its execution at any point, ship itself to a new location on a network, and continue execution on the new machine. On each host, the mobile agent interacts with stationary service agents and other resources to accomplish its task. Mobile agents are goal-oriented, can communicate with other agents, and can continue to operate even after the machine that launched them has been removed from the network.

Mobile agents have several advantages over the traditional client/server model. Experiments show that mobile agents can, among other things, lead to faster applications, reduced bandwidth demands, or less dependence on a reliable network connection. Although none of these benefits are unique to mobile agents, no competing technique shares all of them. The mobile-agent system provides a single general framework in which a wide range of distributed applications can be implemented in an efficient and easy manner.

Mobile agent applications are currently being developed by industry, government, and academia for use in such areas as telecommunications systems, personal digital 
assistants, information management, on-line booking, contract negotiation, air traffic control, parallel processing, and computer simulation.

However, the mobile agent computing paradigm poses several privacy and security challenges, which clearly are one of the main obstacles to the widespread use and adaptation of this new technology. Mobile agent security issues include: authentication, identification, secure messaging, certification, trusted third parties, non-repudiation, and resource control. Mobile agent frameworks must be able to counter new threats as agent hosts must be protected from malicious agents, agents must be protected from malicious hosts, and agents must be protected from malicious agents. Therefore, the next group of papers is directed towards evaluating existing mobile agent security mechanisms and developing new countermeasures for mobile agent security threats.

In the first paper in this group, Raj Gururajan studies security of transactions in mobile commerce. He argues that while most of the various security procedures are limited to corporate IT infrastructure, in mobile commerce issues concerned with transaction security appear to have extended beyond the corporate network to embrace the complete business process. Any lapse in procedures that maintain confidentiality of data or violation of privacy could affect corporate image. In addition to existing security problems in a wired commerce environment, the emergence of mobile devices has renewed calls for addressing security threats to financial transactions. These are the problems discussed in Gururajan's paper as key issues in terms of organisation's architectural and procedural approaches to security, reliability and availability of transactions.

Since all the information about a mobile agent is transported over the Internet, the security policies become very important. However, as Iuon-Chang Lin, Hsia-Hung $\mathrm{Ou}$ and Min-Shiang Hwang point out, the transportation security is usually neglected. Therefore, their paper proposes two secure transportation schemes for mobile agents. These new transportation mechanisms can prevent all possible attacks during the process of transporting agents. Furthermore, users can choose the best transportation scheme according to the system's scale.

\section{Coalition Operations Planning and Negotiations}

Military coalitions are examples of large-scale, multi-faceted, virtual organizations, which sometimes need to be rapidly created and flexibly changed as circumstances change. The agent-based paradigm is a good mechanism of building complex software systems in general, and hence offers potential benefits in the coalition context. 
The next group of papers in the special issue aims to show that multi agent systems are an effective way of dealing with the complexity of real-world problems, such as agile and robust coalition operations and enabling interoperability between heterogeneous components, including legacy and actual military systems.

In the first paper, Zakaria Maamar, Nabil Sahli, Bernard Moulin, and Paul Labbé present their software agent-based collaborative approach to humanitarian-assistance scenarios. Their research deals with the use of software agents as a support to collaboration. Collaboration can face different constraints, such as partner distribution and resource heterogeneity. To deal with these obstacles, the authors propose coordination strategies. They enable agents to avoid conflicts and, hence, to fulfill their activities efficiently. Their work is applied to medical evacuation scenario, in which different participants, such as non-governmental organizations, have to work together despite their individual differences. The authors demonstrate that the agent-based computing paradigm offers a promising new approach to dealing with the technical and non-technical issues in establishing a coherent collaboration environment in humanitarian assistance scenarios.

Coalitions exhibit the problems of integrating single-service and joint capabilities into a coherent force. Furthermore, the nature of a coalition context implies some need to rapidly configure own or foreign systems into a cohesive whole. Many problems in this environment can only be solved by organizational changes and by aligning doctrine, concepts of operations and procedures. Due to the absence of pre-existing coordinated systems, coalition scenarios require a rapid, flexible, on-the-fly approach that allows capabilities to be assembled at run-time. In ensuring interoperability, it is also crucial to address issues of security of data, control over semi-trusted software from other coalition partners, and robustness of the resulting system. Coalition operations are often characterized by data overload, lack of information, labor intensive collection and coordination of information, and standalone command and control systems that use incompatible data formats.

The paper by Zakaria Maamar and Paul Labbé shows that the agent-based computing paradigm offers a promising new approach to dealing with the described issues by embracing the distributed, heterogeneous, and dynamic nature of the coalition environment. The agent-based technologies aim at managing the coalition informational infrastructure, in terms of autonomy, adaptability, and scalability. To develop their agent-based approach to support of coalition operations, the authors identify different aspects of a coalition. These include the coalition structure; the roles and responsibilities held by people within the coalition; the flow of information within the coalition; the capabilities required or available within the coalition; and the context in which the coalition operates. For many of these aspects, software agents can be used. The authors have shown a number of disparate agent systems working 
together in a realistic coalition environment and indicated the value of the agentbased computing paradigm for rapidly creating such agent organizations. Their conclusion is that software agents, together with agent-based infrastructures and services could play a key role in supporting coalition operations. This technology will provide the ability to bring together and integrate legacy or previously incompatible systems quickly to aid in all aspects of coalition operations, without sacrificing security and control. Thus an agent-enabled environment helps create shared understanding and improves the situational awareness of military commanders. Moreover, it could make a significant contribution to the aims of Network-Centric Warfare.

The issue of integrating information systems in coalition operations is further dwelled upon in the contribution by Marvin "Lenard" Simpson, Jr. The author from the US examines in detail the challenges of command and control in a coalition air operation under the presumption that the operation is led by the United States. Being far from standard, the arrangement of a Coalition Aerospace Operations Center, or CAOC, is still a subject of deliberation. We believe that the description in this article will provide to multi-agent researchers a glimpse at a potential testbed for implementation of advanced agent-based technologies.

Recently, there has been an increased interest in the application of the agent-based technology in negotiation. In their paper, Javier Carbo, José Molina and Jorge Davila present a scheme for multi-agent argumentative negotiation in the generic domain encompassing intelligence agencies and informers. The goal of the negotiation phase in their work is to persuade the other party by argumentative reasons. They have studied several typical arguments, which may be useful in this context, such as ultimatums, promises of future fidelity, past behaviour, and future proposals received. Their approach tries to make the negotiation dialogue more human through arguments commonly used in real-life negotiations. They have also studied how to prove certain knowledge without revealing the corresponding details, and how to preserve anonymity during negotiations when arguments such as past payoffs and offerings from other intelligence agencies are available. All this, according to the authors, can be accomplished through the exchange of arguments in counterproposal attributes and with the help of encryption techniques.

\section{Agents in Resource Planning}

Among the many appealing features of the multi-agent technology, its capabilities for collaboration and adaptation are particularly attractive to the military domain. Agents are capable of cooperating and collaborating with other agents and possibly human users to solve problems. Agents share information, knowledge, and tasks among 
themselves, and cooperate with each other to achieve common goals. The attractiveness of the multi-agent system is not only reflected by the intelligence of individual agents but also by the emergent behavior of the entire agent society.

The system developed by David Camacho, José M.Molina, Daniel Borrajo, and Ricardo Aler demonstrates many of the appealing features of the agent-based technology. Their paper presents MAPWEB (Multi-Agent Planning on the Web), a multi-agent system for cooperative work between different intelligent software agents that solve user planning problems using the information stored in the World Wide Web. MAPWEB is a heterogeneous system of intelligent agents whose main characteristics are cooperation, reasoning, and knowledge sharing. The architecture of MAPWEB uses four types of agents: user agents that are the bridge between the users and the system; control agents that are responsible for managing the rest of the agents; planning agents that are able to solve planning problems; and, finally, web agents whose objectives are to retrieve, represent, and share information obtained from the Web. MAPWEB solves planning problems by means of cooperation between planning agents and web agents. Instead of using the planning agents to solve the whole planning problem, they focus on a less restricted problem and cooperate with the web agents to validate and complete abstract solutions. To enable efficient cooperation, the authors define a common language and data structures.

Military logistics planning involves the efficient coordination of supply requests and shares many features common to complex problem situations including information overload, uncertainty, and layers of time constraints. Decision makers coordinate supply orders and determine the availability of requested items, the location that will receive the items, and how the items will reach the appropriate location in the most efficient and timely manner.

The agent-based approach to military logistics planning suggested by Stanimir Stojanov, Roumen Venkov and Radi Radev allows their applications to react quickly to changing logistic requirements and offers technical solutions to complex problems. Their approach to system design incorporates collaborative agents with knowledge in specific domains. These agents create a partnership and collaborate with expert human staff members during the various stages of the logistic process. In addition to creating realistic and timely solutions, the system also monitors the execution of logistic plans and offers real-time decision solutions to complex problems. Their system, DIANA, is a software "tool kit" that can be adapted to the user, to the logistic support concept, and to the changing circumstances of a military contingency. This is an agent-based system that is based on the MALINA-technology. The goal of the authors has been to create a general technological framework for development of modern information systems for military logistics and e-commerce. 
Finally, this special issue provides a comprehensive, up-to-date list with on-line resources on general multi-agent research and journals, security and defense oriented research, projects, and software tools, as well as some milestone publications.

Information \& Security 